'Laboratorio de Investigación en Ciencias Biomédicas. Departamento de Ciencias Básicas. Universidad Católica de la Santísima Concepción. Concepción, Chile. ${ }^{2}$ Institute of Health and Wellbeing. University of Glasgow, Glasgow, United Kingdom. ${ }^{3}$ BHF Glasgow Cardiovascular Research Centre, Institute of Cardiovascular and Medical Sciences, University of Glasgow. Glasgow, United Kingdom.

${ }^{4}$ Instituto de Anatomía, Histología y Patología, Facultad de Medicina, Universidad Austral de Chile. Valdivia, Chile.

${ }^{5}$ Centro de Investigación en Fisiología del Ejercicio (CIFE), Universidad Mayor. Santiago, Chile.

${ }^{6}$ Laboratorio de Rendimiento Humano, Grupo de Estudio en Educación, Actividad Física y Salud (GEEAFyS), Universidad Católica del Maule. Talca, Chile. ${ }^{7}$ Instituto de Farmacia, Facultad de Ciencias, Universidad Austral de Chile. Valdivia, Chile. aBioquímico.

${ }^{\text {b} D o c t o r ~ e n ~ C i e n c i a s ~ B i o l o ́ g i c a s . ~}$ 'Nutricionista, MSc. Nutrición Humana. dProfesora de Biología y Química. MSc. Neurociencias y Salud Mental.

eProfesor de Educación Física. Doctor en Ciencias Cardiovasculares y Biomédicas. ${ }^{f} \mathrm{MSc}$. Nutrición y Dietética.

Trabajo financiado por CONICYT. Los autores declaran no tener conflictos de interés.

Recibido el 23 de octubre de 2019, aceptado el 3 de julio de 2020 .

Correspondencia a: María Adela Martínez-Sanguinetti Instituto de Farmacia, Facultad de Ciencias, Universidad Austral de Chile. Valdivia, Chile. mmartin3@uach.cl

\section{Consumo de azúcares totales y su asociación con obesidad en población chilena - Resultados del estudio GENADIO}

\author{
LORENA MARDONES ${ }^{1, a, b}$, MARCELO VILLAGRÁN ${ }^{1, a, b}$, \\ FANNY PETERMANN-ROCHA ${ }^{2,3, c}$, ANA MARÍA LEIVA ${ }^{4, d}$, \\ CARLOS CELIS-MORALES ${ }^{2,3,5,6, \mathrm{e}}$, \\ MARÍA ADELA MARTÍNEZ-SANGUINETTI ${ }^{7, \mathrm{a}, \mathrm{f}}$, \\ en representación del Consorcio de Investigación ELHOC \\ (Epidemiology of Lifestyle and Health Outcomes in Chile)
}

\section{Total sugar consumption and its association with obesity in Chilean adults}

Background: Sugar intake is a risk factor for the development of obesity. Aim: To investigate the association between total sugar intake and obesity markers in Chilean adults. Material and Methods: This cross-sectional study included 396 participants of the GENADIO study. Anthropometric measurements (body weight, body mass index (BMI), waist circumference (WC), hip circumference (HC), waist-to-hip ratio, and $\%$ body fat) were measured through standardized protocols. Total sugar intake was determined from a 7-days weighted food daily. Sugar intake expressed in g/day was categorized into quartiles. Results: Higher sugar intake was associated with a higher BMI, WC and HC but not with body fat \%. One quartile increment in sugar intake was associated with $1.50 \mathrm{~kg}$ [95\% confidence interval (CI): 0.49; 2.50] higher body weight, $0.34 \mathrm{~kg} / \mathrm{m}^{2}$ [95\% CI: 0.01; 0.69] higher BMI, $1.23 \mathrm{~cm}$ [95\% CI: 0.11; 2.34] higher WC, $1.39 \mathrm{~cm}$ [95\% CI: 0.28; 2.50] higher HC and 0.02 the waist-to-hip ratio [95\% C: 0.01; 0.02]. Conclusions: Total sugar intake was associated with higher adiposity levels in these Chilean adults.

(Rev Med Chile 2020; 148: 906-914)

Key words: Adiposity; Diet; Obesity; Sucrose; Sugars.
L a obesidad es un factor de riesgo para el desarrollo de diversas patologías crónicas no transmisibles, representando la segunda causa de morbi-mortalidad a nivel mundial ${ }^{1}$. Según la última Encuesta Nacional de Salud, $74,2 \%$ de la población adulta presenta obesidad o sobrepeso en Chile 2 .

Una alimentación no saludable junto con un consumo excesivo de energía, son algunos de los factores de riesgo claves en el desarrollo de la obesidad $^{3,4}$. Si bien diferentes macronutrientes se han asociado a obesidad, como lo son el consumo excesivo de grasas y carbohidratos ${ }^{4}$, en la última década se ha puesto especial énfasis al rol que juega el consumo de azúcares en la pandemia de obesidad ${ }^{5-7}$. Muchos estudios han propuesto que el consumo de azúcares totales se asocia con mayor nivel de peso corporal y adiposidad ${ }^{4,6}$, postulándose que esta 
relación está mediada por el aporte de las llamadas "calorías vacías"; sin embargo, hay evidencia que sugiere que la asociación con adiposidad es diferente según el tipo de azúcares consumido ${ }^{4}$.

Los azúcares simples, son carbohidratos de absorción rápida, que provocan aumentos agudos de la glicemia e insulinemia ${ }^{8}$. Los principales azúcares simples consumidos por la población mundial son los monosacáridos glucosa y fructosa y los disacáridos sacarosa, lactosa y maltosa. Estos azúcares se consumen preferentemente como azúcares añadidos en alimentos procesados y en menor proporción, desde fuentes naturales, como leche y frutas 9 . El consumo de alimentos procesados con azúcares añadidos es uno de los principales factores de riesgo para desarrollar obesidad $^{10}$. Si bien la Food and Drug Association (FDA) recomienda un consumo de 50 g/día de azúcares totales (simples), el consumo promedio a nivel mundial es de $73 \mathrm{~g} /$ día y en Chile asciende a 158,6 g/día; datos que ubican a nuestro país en el segundo lugar en el consumo de estos azúcares a nivel mundial ${ }^{11}$.

Chile cursa actualmente una etapa de post-transición nutricional caracterizada por alto consumo de azúcares e incumplimiento de las recomendaciones de alimentación saludable ${ }^{11}$. Teniendo en cuenta la alta tasa de prevalencia de obesidad y de consumo de azúcares en Chile ${ }^{2,12}$, el objetivo de este estudio fue investigar la asociación entre el consumo de azúcares totales y marcadores de obesidad en población adulta chilena.

\section{Materiales y Métodos}

Este trabajo es un estudio descriptivo transversal que incluyó a 396 individuos pertenecientes al estudio GENADIO (Genes, Ambiente, Diabetes y Obesidad) y que contaban con información relacionada con el consumo de azúcares totales y marcadores de adiposidad. El proyecto GENADIO fue realizado en Chile entre los años 2009-2011, con el objetivo de evaluar la prevalencia de factores de riesgo de enfermedades cardiovasculares ${ }^{13}$. La población estudiada incluyó a personas no indígenas e indígenas (Mapuches), que habitaban en zonas rurales (Ralco, Neltume, Panguipulli) y urbanas (Concepción, Temuco, Valdivia). Las personas incluidas no presentaban historial médico de enfermedad metabólica o cardiovascular, y al momento de la evaluación no recibían prescripción de medicamentos ${ }^{14}$. Se contó con la aprobación de los Comités de Ética de la Universidad de Chile, Universidad de Concepción y Universidad de Glasgow. Todos los participantes firmaron su consentimiento informado previo a la recolección de datos.

\section{Variables antropométricas y marcadores de adiposidad}

La evaluación antropométrica se realizó por personal capacitado utilizando protocolos estandarizados. El peso y talla corporal fueron determinados con una balanza electrónica (TANITA TBF 300A, USA) y tallímetro (SECA A800, USA), con una precisión de $100 \mathrm{~g}$ y $1 \mathrm{~mm}$, respectivamente. El perímetro de cintura (PC) y el perímetro de cadera fueron medidos con cinta métrica no distensible (SECA Modelo 201, USA). El estado nutricional fue clasificado en base a los puntos de corte sugeridos por la Organización Mundial de la Salud (OMS) para el índice de masa corporal (IMC): bajo peso: $<18,5 \mathrm{~kg} / \mathrm{m}^{2}$; normopeso: $18,5-24,9 \mathrm{~kg} /$ $\mathrm{m}^{2}$; sobrepeso: $25,0-29,9 \mathrm{~kg} / \mathrm{m}^{2}$ y obesidad: $\geq 30,0$ $\mathrm{kg} / \mathrm{m}^{2(11,15)}$. Los valores utilizados para definir obesidad central fueron $P C \geq 102 \mathrm{~cm}$ y $\geq 88 \mathrm{~cm}$, en hombres y mujeres, respectivamente ${ }^{13}$. La composición corporal se determinó mediante la relación cintura/cadera y la medición de cuatro pliegues cutáneos (bicipital, sub-escapular, supra-iliaco y tricipital), a través de un cáliper Harpenden skinfold (Cranlea \& Company, Birmingham, UK). Se aplicó la ecuación de Durnin y Womersley para estimar el porcentaje de masa grasa corporal ${ }^{16}$.

\section{Ingesta energética, de macronutrientes y alcohol}

La ingesta dietaria fue evaluada a través del registro de 7 días del consumo de bebidas y alimentos, los cuales fueron pesados previo a su consumo en una balanza de cocina marca Seca y que fue entregada a cada participante por los investigadores del estudio ${ }^{13}$. La ingesta dietaria fue analizada por la Base de Datos de Composición de Alimentos de Chile a través del Software MINUTA de la Universidad de Concepción. Para cada participante se obtuvieron datos de consumo de energía, macronutrientes y alcohol. El consumo de azúcares totales fue clasificado en cuatro grupos: cuartil 1: $<74 \mathrm{~g} /$ día; cuartil 2: entre $74-112$ g/día; cuartil 3: entre 113-178 g/día y cuartil 4: $>178$ g/día. Los azúcares totales, incluyó a todos los azúcares simples (monosacáridos + disacári- 
dos). Se consideran los azúcares simples libres o añadidos durante la elaboración (caramelos, chocolates, pasteles, bebidas azucaradas, etc.) más los azúcares simples naturales o intrínsecos (presentes en frutas, verduras, leche, etc. $)^{17,18}$.

\section{Variables sociodemográficas y de estilo de vida}

Los datos sociodemográficos (género, edad, ingresos, etnia, lugar de residencia [urbano/rural] y nivel educacional) y estilo de vida (consumo de tabaco y alcohol), fueron recolectados mediante encuestas validadas ${ }^{13}$. Para la selección de participantes de ascendencia mapuche o europea y el descarte de individuos mestizos, se incluyeron solo aquellos cuyos apellidos paterno y materno fuesen de origen mapuche o europeo, respectivamente. Además, para la selección de mapuches, se descartaron aquellos cuyo grupo sanguíneo fuese distinto al grupo O. Los niveles de actividad física (AF) y el tiempo sedentario fueron estimados utilizando acelerómetro de movimiento (Actigraph GTM1, USA). La intensidad de la AF y el gasto energético se determinaron mediante el algoritmo de Freedson ${ }^{19}$.

\section{Análisis estadístico}

Los datos de caracterización de la población estudiada son presentados como promedio y desviación estándar (DE) para variables continuas y como porcentaje para variables categóricas.

La asociación entre el consumo de azúcares totales y marcadores de obesidad (peso corporal, IMC, PC, perímetro de cadera, relación cintura/ cadera, \% masa grasa corporal), se realizó utilizando análisis de regresión lineal, representando los datos obtenidos como promedio y sus respectivos intervalos de confianza (95\% IC). La asociación entre obesidad, obesidad central y cuartiles de consumo de azúcares totales fue determinada por análisis de regresión logística, representando los datos obtenidos como odds ratio (OR) y sus respectivos 95\% IC, usando como referencia el cuartil de menor consumo de azúcares.

Todos los análisis fueron ajustados por variables de confusión mediante la utilización de tres modelos estadísticos. Modelo 0: sin ajustar; Modelo 1: ajustado por edad, género, etnia, nivel educacional y lugar de residencia; y Modelo 2: ajustado por el Modelo 1 y AF. Para todos los análisis se utilizó el programa STATA SE v14. El nivel de significancia fue definido como $\mathrm{p}<0,05$.

\section{Resultados}

En la Tabla 1 se presentan las características generales de la población según cuartil de consumo de azúcares totales. La mayor diferencia entre los distintos cuartiles se presentó en el estado nutricional y en la ingesta calórica. El cuartil más alto presentó mayor porcentaje de individuos con estado nutricional alterado (87,9\% con sobrepeso u obesidad) y una ingesta calórica $45 \%$ mayor a los cuartiles 1 y 2 (3.456 vs. $2.372 \mathrm{Kcal} /$ día). También se observó que en el cuartil de mayor ingesta de azúcares totales existió mayor proporción de hombres $(61,6 \%)$, de personas con menor nivel educacional $(87,9 \%)$ y de etnia mapuche $(76,8 \%)$.

Los resultados presentados en la Tabla 2 revelan una asociación positiva entre la ingesta de azúcares totales y los distintos marcadores de obesidad, con excepción del \% de masa grasa corporal. Esta asociación se mantuvo incluso en el modelo más ajustado, el cual incluyó variables sociodemográficas y de AF (Modelo 2). En el modelo sin ajustar (Modelo 0), el peso corporal aumentó $1,30 \mathrm{~kg}$ por cada 1 cuartil de incremento en el consumo de azúcares totales (95\% IC: 0,41; 2,18), el IMC 0,50 kg/m² (95\% IC: $1,90 ; 0,82), 2,20 \mathrm{~cm}$ el PC (95\% IC: 1,$23 ; 2,16)$, $1,90 \mathrm{~cm}$ el perímetro de cadera (95\% IC: 0,96 ; $2,84)$ y 0,02 la relación cintura/cadera (95\% IC: 0,$01 ; 0,03$ ). En el modelo más ajustado (Modelo 2 ), las asociaciones disminuyeron levemente su magnitud, pero permanecieron significativas. El peso corporal aumentó $1,50 \mathrm{~kg}(95 \%$ IC 0,49 ; 2,50 ), el IMC $0,34 \mathrm{~kg} / \mathrm{m}^{2}$ (95\% IC 0,01; 0,69), el perímetro cintura $1,23 \mathrm{~cm}(95 \%$ IC 0,$11 ; 2,34)$, el perímetro de cadera $1,39 \mathrm{~cm}$ (95\% IC 0,29; $2,39)$ y la relación cintura/cadera 0,02 (95\% IC $0,01 ; 0,02)$ (Figura 1).

La asociación entre los cuartiles de consumo de azúcares totales y la probabilidad de ser obeso o tener obesidad central se presentan en la Tabla 3. No se observó asociación entre consumo de azúcares totales y obesidad, determinada por IMC para ninguno de los modelos estadísticos. Para obesidad central, se observó una asociación con el consumo de azúcares totales solamente para el modelo ajustado por variables sociodemográficas (OR: 1,36 [95\% IC: 1,06; 1,76]); sin embargo, al ajustar este modelo y además por AF, esta asociación desapareció (OR: 1,09 [95\% IC: 0,83; 1,44]) (Tabla 3). 
Tabla 1. Características de los participantes según consumo de azúcares totales

\begin{tabular}{|c|c|c|c|c|}
\hline & \multicolumn{4}{|c|}{ Cuartil de consumo de azúcares totales } \\
\hline & Bajo & Medio/Bajo & Medio/Alto & Alto \\
\hline $\mathrm{n}$ & 99 & 99 & 99 & 99 \\
\hline Edad (años) & $40,0 \pm 13,0$ & $36,1 \pm 13,5$ & $35,812,0$ & $37,0 \pm 13,3$ \\
\hline Género, \% hombres & 23,2 & 54,5 & 48,6 & 61,6 \\
\hline \multicolumn{5}{|l|}{ Nivel educacional (\%) } \\
\hline Nivel básico & 24,2 & 14,3 & 35,4 & 49,5 \\
\hline Enseñanza media & 40,4 & 54,1 & 31,3 & 38,4 \\
\hline Técnico/universitario & 35,3 & 31,6 & 33,3 & 12,1 \\
\hline \multicolumn{5}{|l|}{ Ingresos (\%) } \\
\hline Bajo & 33,67 & 34,02 & 47,47 & 66,67 \\
\hline Medio & 12,24 & 14,43 & 9,09 & 9,09 \\
\hline Alto & 54,08 & 51,55 & 43,43 & 24,24 \\
\hline Zona geográfica urbana, \% & 52,53 & 70,71 & 43,43 & 34,34 \\
\hline Etnia Mapuche (\%) & 30,30 & 42,42 & 53,54 & 76,77 \\
\hline Tabaquismo (\%) & 61,6 & 50,5 & 48,5 & 55,6 \\
\hline Peso (kg) & $69,6 \pm 11,0$ & $69,9 \pm 9,4$ & $70,4 \pm 8,8$ & $75,2 \pm 10,0$ \\
\hline IMC $\left(\mathrm{kg} / \mathrm{m}^{-2}\right)$ & $28,3 \pm 3,7$ & $27,2 \pm 3,8$ & $28,1 \pm 3,3$ & $29,0 \pm 3,3$ \\
\hline \multicolumn{5}{|l|}{ Estado nutricional (\%) } \\
\hline Normal & 21,2 & 25,3 & 18,2 & 12,2 \\
\hline Sobrepeso & 35,4 & 20,2 & 24,2 & 35,4 \\
\hline Obeso & 43,4 & 54,6 & 57,6 & 52,5 \\
\hline Masa grasa corporal \% & $29,8 \pm 4,1$ & $29,7 \pm 4,6$ & $29,2 \pm 4,7$ & $29,0 \pm 5,1$ \\
\hline Perímetro de cintura $(\mathrm{cm})$ & $96,4 \pm 0,1$ & $94,8 \pm 10,1$ & $97,5 \pm 10,2$ & $102,1 \pm 10,0$ \\
\hline Obesidad central (\%) & 70,71 & 50,51 & 65,66 & 65,66 \\
\hline \multicolumn{5}{|l|}{ Actividad física } \\
\hline Actividad física (MET.hr-1. sem-1) & $14,2 \pm 4,5$ & $14,2 \pm 4,5$ & $15,2 \pm 5,2$ & $15,4 \pm 5,1$ \\
\hline Tiempo sedentario (min. dia ${ }^{-1}$ ) & $9,0 \pm 1,4$ & $9,0 \pm 1,4$ & $8,4 \pm 1,6$ & $8,8 \pm 1,6$ \\
\hline \multicolumn{5}{|l|}{ Alimentación (\% ET.dia-1) } \\
\hline Proteínas & $16,3 \pm 5,4$ & $16,3 \pm 5,4$ & $13,7 \pm 3,7$ & $12,8 \pm 3,5$ \\
\hline Grasas totales & $23,3 \pm 9,0$ & $23,3 \pm 9,0$ & $28,6 \pm 6,6$ & $26,0 \pm 7,8$ \\
\hline Grasas saturadas & $0,7 \pm 0,4$ & $0,7 \pm 4,0$ & $0,8 \pm 0,3$ & $0,6 \pm 0,3$ \\
\hline Grasas mono-insaturadas & $7,3 \pm 4,0$ & $7,3 \pm 4,0$ & $8,8 \pm 3,8$ & $7,4 \pm 3,3$ \\
\hline Grasas poli-insaturadas & $3,7 \pm 2,2$ & $3,7 \pm 2,1$ & $4,9 \pm 2,5$ & $4,1 \pm 1,8$ \\
\hline Carbohidratos & $58,0 \pm 11,6$ & $58,8 \pm 11,9$ & $55,4 \pm 8,0$ & $58,1 \pm 7,6$ \\
\hline Azúcares simples & $9,6 \pm 4,1$ & $17,6 \pm 4,4$ & $22,3 \pm 5,2$ & $29,4 \pm 6,5$ \\
\hline Alcohol & $1,6 \pm 1,9$ & $1,6 \pm 1,8$ & $2,3 \pm 2,0$ & $3,2 \pm 3,0$ \\
\hline Consumo energético (Kcal.dia-1) & $2.372 \pm 833$ & $2.372 \pm 833$ & $2.700 \pm 557$ & $3.456 \pm 764,7$ \\
\hline
\end{tabular}

Datos presentados como promedio y desviación estándar (DE) para variables continuas y como \% para variables categóricas. Los puntos de corte de los cuartiles de consumo de azúcares totales son: cuartil 1: < 74 g/día; cuartil 2: entre 74 y 112 g/ día; cuartil 3: entre 113 y 178 g/día y cuartil 4: >178 g/día. ET: energía total, MET: equivalente metabólico total, min: minuto, sem: semana. 


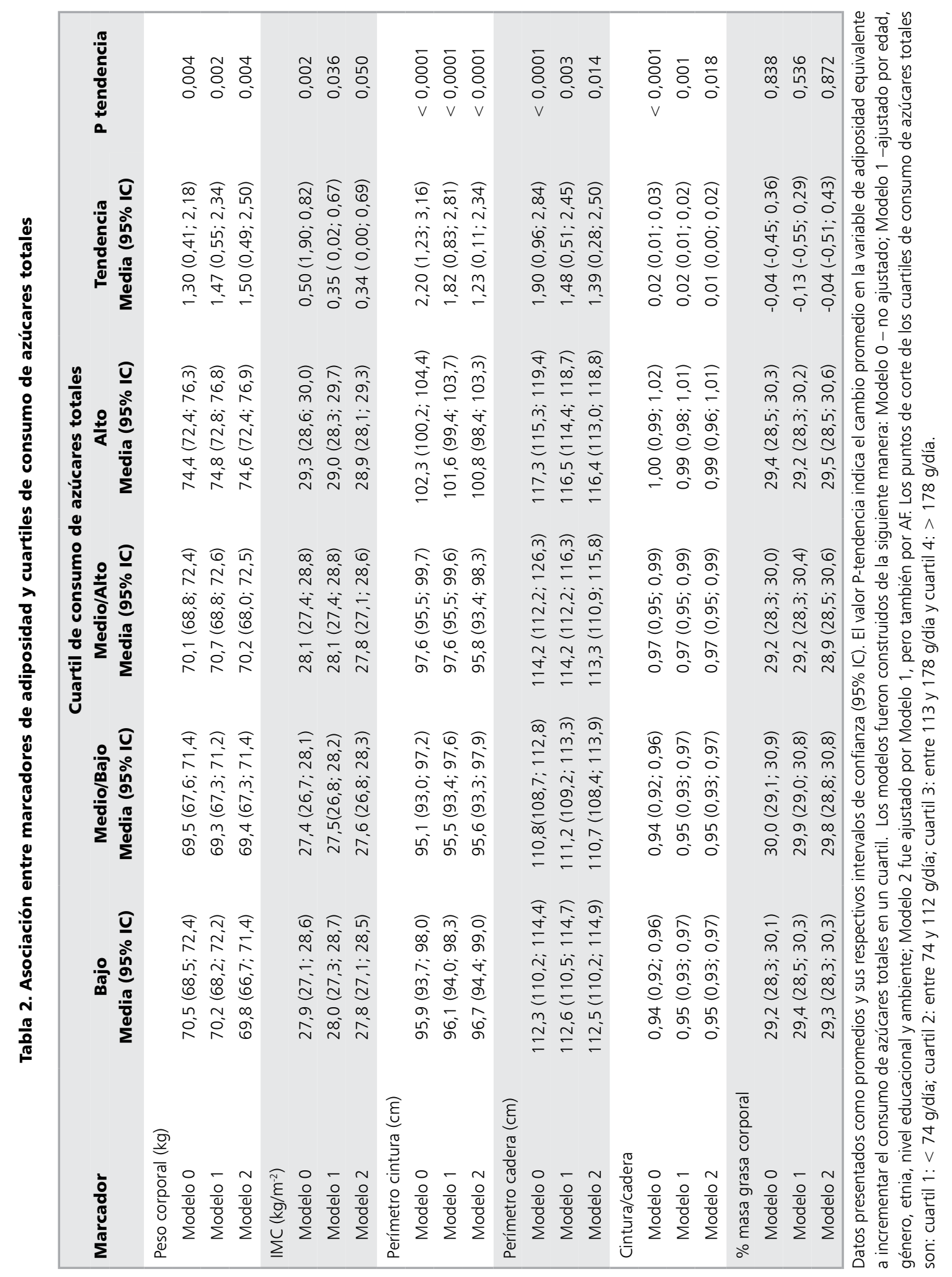



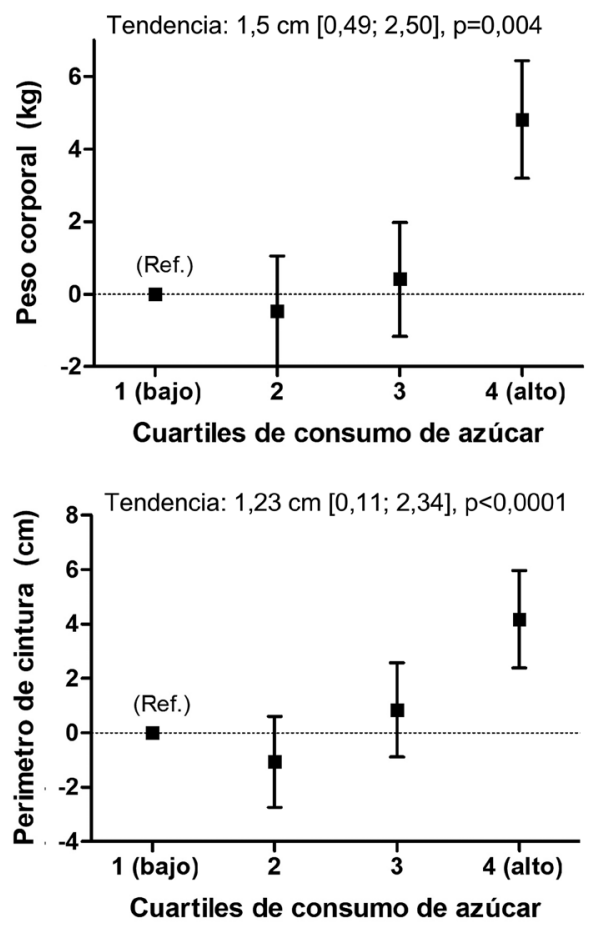
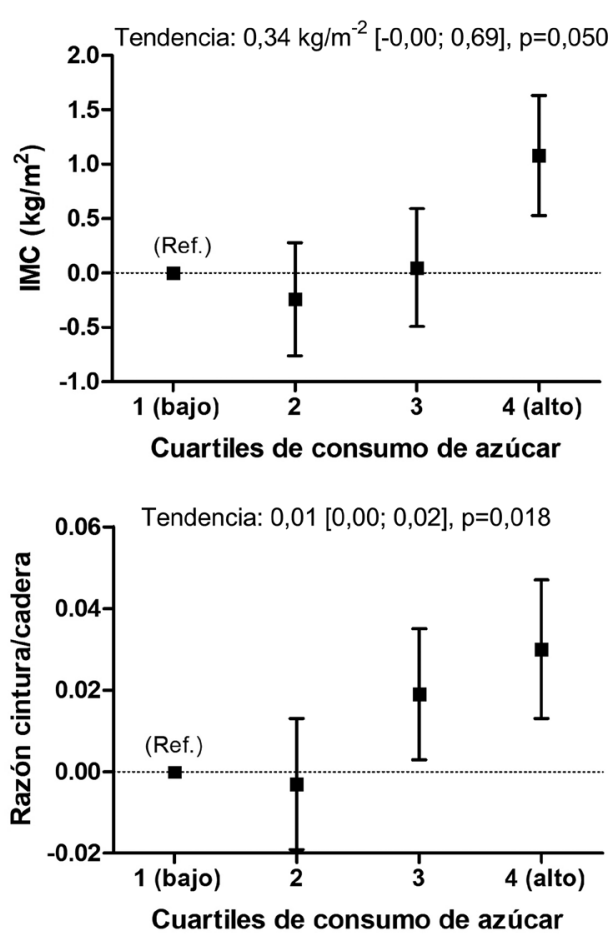

Figura 1. Asociación entre marcadores de adiposidad y consumo de azúcares totales. Datos presentados como el valor tendencia promedio y sus respectivos intervalos de confianza ( $95 \%$ IC) para el modelo más ajustado (modelo 2). El valor tendencia indica el cambio promedio en la variable de adiposidad equivalente a incrementar el consumo de azúcares totales en un cuartil. Los puntos de corte de los cuartiles de consumo de azúcares totales son: cuartil 1: < 74 g/día; cuartil 2: entre 74 y 112 g/día; cuartil 3: entre 113 y $178 \mathrm{~g} /$ día y cuartil 4: >178 g/día.

Tabla 3. Asociación entre niveles de adiposidad y cuartiles de consumo de azúcares totales

\begin{tabular}{|c|c|c|c|c|c|c|}
\hline \multirow[b]{2}{*}{ Marcador } & \multicolumn{6}{|c|}{ Cuartil de consumo de azúcares totales } \\
\hline & $\begin{array}{c}\text { Bajo } \\
\text { OR (95\% IC) }\end{array}$ & $\begin{array}{l}\text { Medio/Bajo } \\
\text { OR (95\% IC) }\end{array}$ & $\begin{array}{l}\text { Medio/Alto } \\
\text { OR (95\% IC) }\end{array}$ & $\begin{array}{c}\text { Alto } \\
\text { OR }(95 \% \text { IC) }\end{array}$ & $\begin{array}{c}\text { Tendencia } \\
\text { OR (95\% IC) }\end{array}$ & $\stackrel{\text { P }}{\text { tendencia }}$ \\
\hline \multicolumn{7}{|l|}{ Obesidad } \\
\hline Modelo 0 & 1,00 (Ref) & $0,48(0,21 ; 1,07)$ & $0,80(0,35 ; 1,81)$ & $1,75(0,75 ; 4,01)$ & $1,20(0,93 ; 1,55)$ & 0,159 \\
\hline Modelo 1 & 1,00 (Ref) & $0,67(0,27 ; 1,67)$ & $1,03(0,41 ; 2,60)$ & $1,85(0,67 ; 5,10)$ & $1,22(0,90 ; 1,67)$ & 0,194 \\
\hline Modelo 2 & 1,00 (Ref) & $1,47(0,46 ; 4,70)$ & $0,30(0,08 ; 1,09)$ & $1,42(0,45 ; 4,51)$ & $1,00(0,70 ; 1,43)$ & 0,984 \\
\hline \multicolumn{7}{|c|}{ Obesidad Central } \\
\hline Modelo 0 & 1,00 (Ref) & $0,42(0,24 ; 0,76)$ & $0,79(0,43 ; 1,44)$ & $0,79(0,43 ; 1,44)$ & $1,00(0,83 ; 1,2)$ & 1,000 \\
\hline Modelo 1 & 1,00 (Ref) & $0,99(0,47 ; 2,11)$ & $1,40(0,66 ; 2,96)$ & $2,52(1,11 ; 5,71)$ & $1,36(1,06 ; 1,76)$ & 0,015 \\
\hline Modelo 2 & 1,00 (Ref) & $1,51(0,62 ; 3,65)$ & $0,96(0,36 ; 2,51)$ & $1,52(0,63 ; 3,67)$ & $1,09(0,83 ; 1,44)$ & 0,362 \\
\hline
\end{tabular}

Datos presentados como odds ratio (OR) y sus respectivos intervalos de confianza (95\% IC). El grupo de referencia es el cuartil con consumo bajo de azúcar. El valor P-tendencia indica el cambio en el OR de la variable de adiposidad equivalente a incrementar el consumo de azúcares totales en un cuartil. Los modelos fueron construidos de la siguiente manera: Modelo 0 - no ajustado; Modelo 1 - ajustado por edad, género, etnia, nivel educacional y ambiente; Modelo 2 - ajustado por Modelo 1, pero también por AF. Los puntos de corte de los cuartiles de consumo de azúcares totales son: cuartil 1: $<74$ g/día; cuartil 2: entre 74 y 112 g/día; cuartil 3: entre 113 y 178 g/día y cuartil 4: > 178 g/día. 


\section{Discusión}

Los resultados de este estudio evidencian una asociación entre marcadores de adiposidad (peso corporal, IMC, PC, perímetro de cadera e índice cintura/cadera) y consumo de azúcares totales, incluso tras ajustar por variables de confusión (variables sociodemográficas y de AF). Se observó un aumento promedio de $1,5 \mathrm{~kg}$ de peso corporal y de $0,34 \mathrm{~kg} / \mathrm{m}^{2}$ en IMC por cada incremento en un cuartil de consumo de azúcares totales, lo que corrobora que el mayor consumo de estos productos se relaciona con un balance energético positivo.

Estudios previos encontraron distintos resultados, según la población evaluada y el tipo de diseño metodológico ${ }^{20,21}$. En un estudio prospectivo realizado en Australia, Hodge y cols. identificaron que un mayor consumo de bebidas azucaradas se asociaba con un mayor perímetro de cadera, sin embargo, investigaciones realizadas en jóvenes y adultos estadounidenses no evidenciaron dicha asociación $^{20,21}$. Por otro lado, Stanhope y Havel, al administrar por 10 semanas una dieta alta en fructosa o glucosa a individuos con obesidad/ sobrepeso ( $25 \%$ de las calorías diarias), observaron un aumento significativo del peso corporal y de la masa grasa en ambos grupos ${ }^{22}$. Además, se determinó que la dieta alta en fructosa se relaciona directamente con dislipidemia y resistencia a insulina $^{22}$. Se sabe que el efecto de las dietas altas en fructosa se ve influenciada por el desarrollo de $\mathrm{AF}$, consumo de alcohol y consumo de grasa ${ }^{8,23,24}$.

La nueva directriz de la OMS sobre la ingesta de azúcares del año 2015 recomienda reducir el consumo de azúcares libres a menos de $10 \%$ de la ingesta energética diaria y por debajo de $5 \%$ para obtener efectos beneficios adicionales para la salud $^{18}$. No obstante, en Chile, más de $50 \%$ de la población supera esta cifra, debido principalmente a un alto consumo de bebidas azucaradas $(328 \mathrm{ml}$ per cápita/día, equivalente a $188 \mathrm{kcal})^{25}$. El alto consumo de estas bebidas se presenta principalmente en estratos socioeconómicos bajos, siendo el tercer producto más importante en la canasta familiar básica, luego de productos de primera necesidad, como carne y pan. Estos resultados evidencian un problema sociocultural en Chile, caracterizado por un aumento del consumo de productos excesivamente endulzados y alimentos no saludables en general ${ }^{26}$.

Considerando que la ingesta de productos altamente energéticos se asocia con el desarrollo de obesidad, en diversos países se han implementado políticas públicas con el fin de disminuir su consumo, entre ellas, el aumento en los impuestos a este tipo de alimentos ${ }^{25}$. En Chile, desde el año 2014 se aplica el impuesto adicional a las bebidas no alcohólicas, aumentando de 13 a 18\% el impuesto para bebidas azucaradas con más de $6,25 \mathrm{~g}$ azúcares totales por $100 \mathrm{ml}$ y reduciendo a $10 \%$ el impuesto a aquellas bebidas con menor cantidad $^{27}$. Esta medida permitió disminuir 21\% el consumo de bebidas azucaradas en nuestro país ${ }^{12,15}$. Por otro lado, el año 2016 se comenzó a aplicar en Chile la ley de etiquetado de alimentos (Ley 20.606), que establece que los alimentos y bebidas poco saludables deberán llevar una señal de advertencia correspondiente a un sello negro en forma de "disco pare" con el texto "alto en sodio", "alto en azúcares", "alto en grasas saturadas" y/o “alto en calorías” en letras blancas, según corresponda. Esta ley se ha implementado en tres etapas, reduciendo el límite permitido en contenido de azúcares totales a 22,5 g/100 g de producto sólido y $6 \mathrm{~g} / 100 \mathrm{ml}$ de alimentos líquidos en su primera etapa (2016) y a $10 \mathrm{~g} / 100 \mathrm{~g}$ de producto sólido y $5 \mathrm{~g} / 100 \mathrm{ml}$ de alimentos líquidos en la etapa actual $(2019)^{28}$. Luego de dos años de vigencia de la primera etapa de esta ley, se ha reducido en $25 \%$ la venta de bebidas altas en azúcares totales y la industria alimentaria ha reducido hasta $35 \%$ el contenido de azúcares en sus productos ${ }^{28,29}$. Otros países, como Reino Unido y Ecuador cuentan con un sistema de semáforo que ha sido efectivo ${ }^{30,31}$. Estudios internacionales estiman que este tipo de intervenciones podrían provocar una disminución de 2,4\% en el consumo de azúcares simples; sin embargo, será necesario esperar algún tiempo para poder cuantificar los efectos de estas medidas en la reducción de la incidencia de obesidad en la población chilena ${ }^{31}$.

\section{Fortalezas y limitaciones del estudio}

Entre las fortalezas de este estudio se encuentra la determinación de la ingesta dietaria de los participantes a través de un protocolo de registro del peso de los alimentos consumidos durante 7 días. Este tipo de instrumento muestra un menor error procedimental que el cuestionario de recordatorio de ingesta. Sin embargo, este estudio no está 
ajeno a limitaciones, una de ellas es el reducido tamaño muestral, junto con el alto porcentaje de individuos con sobrepeso/obesidad dentro de la población estudiada. Estos factores afectan el poder estadístico de la asociación entre consumo de azúcares totales y riesgo de obesidad. Otra limitante es la falta de información sobre las distintas fuentes asociadas al consumo de azúcares, como bebidas azucaradas, helados, golosinas, etc., junto con la falta de información sobre los distintos tipos de azúcares totales consumidos (ej. disacáridos o monosacáridos). Estudios futuros deberían incluir este tipo de información para poder entender en mayor detalle la asociación entre el consumo de azúcares y obesidad en población chilena. Esto cobra aún más relevancia al considerar que el consumo de bebidas azucaradas (no alcohólicas) en Chile representa 6,41\% del aporte calórico diario, mientras que el consumo de frutas (enteras o en jugo) alcanza un valor $4,75 \%$, evidenciando que la principal fuente de consumo de azúcares totales en nuestro país son las bebidas no alcohólicas azucaradas ${ }^{9}$.

\section{Conclusiones}

Los resultados evidencian una asociación positiva entre el consumo de azúcares totales y marcadores de obesidad en población adulta chilena. Dada la estrecha relación existente entre obesidad y el desarrollo de enfermedades crónicas no transmisibles, esta información es consistente con las políticas públicas existentes, visualizándose la necesidad de impulsar el desarrollo de nuevas iniciativas que contribuyan a disminuir la prevalencia de obesidad y patologías asociadas en Chile, por ejemplo, realizar intervenciones para disminuir la habituación al sabor dulce desde edades tempranas.

\section{Referencias}

1. Wiss DA, Avena N, Rada P. Sugar Addiction: From Evolution to Revolution. Front Psychiatry 2018; 9: 545. doi: 10.3389/fpsyt.2018.00545.

2. MINSAL. Encuesta Nacional de Salud 2016-2017 Ministerio de Salud. Chile. Disponible en http://www.minsal. cl/wp-content/uploads/2017/11/ENS-2016-17_PRIMEROS-RESULTADOS.pdf. 2017.
3. Bluher M. Obesity: global epidemiology and pathogenesis. Nat Rev Endocrinol 2019; 15 (5): 288-98. doi: 10.1038/s41574-019-0176-8.

4. Anderson JJ, Celis-Morales CA, Mackay DF, Iliodromiti S, Lyall DM, Sattar N, et al. Adiposity among 132479 UK Biobank participants; contribution of sugar intake vs other macronutrients. Int J Epidemiol 2017; 46 (2): 492-501. doi: 10.1093/ije/dyw173.

5. Bray GA, Popkin BM. Dietary sugar and body weight: have we reached a crisis in the epidemic of obesity and diabetes?: health be damned! Pour on the sugar. Diabetes Care 2014; 37 (4): 950-6. doi: 10.2337/dc13-2085.

6. Te Morenga L, Mallard S, Mann J. Dietary sugars and body weight: systematic review and meta-analyses of randomised controlled trials and cohort studies. BMJ. 2012; 346: e7492. doi: 10.1136/bmj.e7492.

7. Swithers SE, Shearer J. Obesity: Sweetener associated with increased adiposity in young adults. Nat Rev Endocrinol 2017; 13 (8): 443-4. doi: 10.1038/nrendo.2017.71.

8. Carvallo P, Carballo E, Barbosa-da-Silva S, Mardarim-de-Lacerda CA, del Sol M. (NAFLD and High Fructose Intake. A Review of Literature). Int J Morphol 2017; 35 (2): 676-83.

9. Kovalskys I, Fisberg M, Gómez G, Pareja RG, Yepez García MC, Cortes Sanabria LY, et al. Energy intake and food sources of eight Latin American countries: results from the Latin American Study of Nutrition and Health (ELANS). Public Health Nutr 2018; 21 (14): 2535-47. doi: 10.1017/S1368980018001222.

10. Hall KD, Ayuketah A, Brychta R, Cai H, Cassimatis T, Chen KY, et al. Ultra-Processed Diets Cause Excess Calorie Intake and Weight Gain: An Inpatient Randomized Controlled Trial of Ad Libitum Food Intake. Cell Metab 2019; 30 (1): 226. doi: 10.1016/j.cmet.2019.05.020.

11. Nakamura R, Mirelman AJ, Cuadrado C, Silva-Illanes N, Dunstan J, Suhrcke M. Evaluating the 2014 sugar-sweetened beverage tax in Chile: An observational study in urban areas. Plos Med 2018; 15 (7): e1002596. doi: 10.1371/journal.pmed.1002596.

12. Bascunan J, Cuadrado C. Effectiveness of sugar-sweetened beverages taxes to reduce obesity: evidence brief for policy. Medwave 2017; 17 (8): e7054. doi: 10.5867/ medwave.2017.08.7054.

13. Celis-Morales CA, Pérez-Bravo F, Ibanes L, Sanzana R, Hormazabal E, Ulloa N, et al. Insulin resistance in Chileans of European and indigenous descent: evidence for an ethnicity x environment interaction. PLoS One 2011; 6 (9): e24690. doi: 10.1371/journal.pone.0024690.

14. Celis-Morales C, Marsaux CF, Livingstone KM, Navas-Carretero S, San-Cristóbal R, Fallaize R, et al. Can genetic-based advice help you lose weight? 
Findings from the Food4Me European randomized controlled trial. Am J Clin Nutr 2017; 105 (5): 1204-13. doi: 10.3945/ajcn.116.145680.

15. WHO. Obesity: preventing and managing the global epidemic.: World Health Organization; (cited 2019 July 20th). Available from: http://www.who.int/nutrition/ publications/obesity/WHO_TRS_894/en/

16. Durnin JV, Womersley J. Body fat assessed from total body density and its estimation from skinfold thickness: measurements on 481 men and women aged from 16 to 72 years. Br J Nutr 1974; 32 (1): 77-97.

17. Rodríguez Delgado J. Azúcares... ¿los malos de la dieta? Rev Pediatr Aten Primaria Supl 2017; 26: 69-75.

18. WHO. Guideline: Sugars intake for adults and children. Geneva: World Health Organization; 2015. Disponible en: https://www.who.int/publications/i/ item/9789241549028.

19. Freedson PS, Melanson E, Sirard J. Calibration of the Computer Science and Applications, Inc. accelerometer. Med Sci Sports Exerc 1998; 30 (5): 777-81. doi: 10.1097/00005768-199805000-00021.

20. Hodge AM, Bassett JK, Milne RL, English DR, Giles GG. Consumption of sugar-sweetened and artificially sweetened soft drinks and risk of obesity-related cancers. Public Health Nutr 2018; 21 (9): 1618-26. doi: 10.1017/ S1368980017002555.

21. Sun SZ, Anderson GH, Flickinger BD, Williamson-Hughes PS, Empie MW. Fructose and non-fructose sugar intakes in the US population and their associations with indicators of metabolic syndrome. Food Chem Toxicol 2011; 49 (11): 2875-82. doi: 10.1016/j.fct.2011.07.068.

22. Stanhope KL, Havel PJ. Fructose consumption: recent results and their potential implications. Ann N Y Acad Sci 2010; 1190: 15-24. doi: 10.1111/j.17496632.2009.05266.x.

23. Rattanavichit Y, Buniam J, Surapongchai J, Saengsirisuwan V. Voluntary exercise opposes insulin resistance of skeletal muscle glucose transport during liquid fructose ingestion in rats. J Physiol Biochem 2018; 74 (3): 455-66. doi: 10.1007/s13105-018-0639-8.

24. Rizkalla SW. Health implications of fructose consumption: A review of recent data. Nutr Metab (Lond) 2010; 7: 82. doi: 10.1186/1743-7075-7-82.

25. Sassi F, Belloni A, Mirelman AJ, Suhrcke M, Thomas A, Salti N, et al. Equity impacts of price policies to promote healthy behaviours. Lancet 2018; 391 (10134): 2059-70. doi: 10.1016/S0140-6736(18)30531-2.

26. Guerrero-López CM, Unar-Munguia M, Colchero MA. Price elasticity of the demand for soft drinks, other sugar-sweetened beverages and energy dense food in Chile. BMC Public Health 2017; 17 (1): 180. doi: 10.1186/ s12889-017-4098-x.

27. Cediel G, Reyes M, da Costa Louzada ML, Martínez Steele E, Monteiro CA, Corvalán C, et al. Ultra-processed foods and added sugars in the Chilean diet (2010). Public Health Nutr 2018; 21 (1): 125-33. doi: 10.1017/ S1368980017001161.

28. Riveros MJ, Parada A, Pettinelli P. [Fructose consumption and its health implications; fructose malabsorption and nonalcoholic fatty liver disease]. Nutr Hosp 2014; 29 (3): 491-9. doi: 10.3305/nh.2014.29.3.7178.

29. Correa T, Fierro C, Reyes M, Dillman Carpentier FR, Taillie LS, Corvalan C. Responses to the Chilean law of food labeling and advertising: exploring knowledge, perceptions and behaviors of mothers of young children. Int J Behav Nutr Phys Act 2019; 16 (1): 21. doi: 10.1186/ s12966-019-0781-x.

30. Lustig RH, Schmidt LA, Brindis CD. Public health: The toxic truth about sugar. Nature 2012; 482 (7383): 27-9. doi: 10.1038/482027a.

31. Petermann F, Leiva AM, Martínez MA, Salas C, Garrido-Méndez A, Celis-Morales C. (Consumo de bebidas azucaradas ayer y hoy: ¿Cuál es el escenario para la población chilena?). Rev Chil Nutr 2017; 4 (44): 400-1. 